\title{
Non-Rodent Model
}

National Cancer Institute

\section{Source}

National Cancer Institute. Non-Rodent Model. NCI Thesaurus. Code C19300.

Research animal models that do not include mice, rats, etc, but that might include nonhuman primates, cats, dogs, and others. 